\title{
High GOLPH3 expression is associated with poor prognosis and invasion of hepatocellular carcinoma
}

\author{
QIAN LI ${ }^{1}$, YUWEN MA ${ }^{1}$ and WANPENG XU ${ }^{2}$ \\ Departments of ${ }^{1}$ Hepatobiliary Surgery and ${ }^{2}$ Anorectal Surgery, \\ Affiliated Hospital of Weifang Medical University, Weifang, Shandong 261031, P.R. China
}

Received February 26, 2014; Accepted December 2, 2014

DOI: $10.3892 / \mathrm{mmr} .2015 .3267$

\begin{abstract}
Golgi phosphoprotein 3 (GOLPH3) overexpression has previously been associated with the progression of several solid tumors, which resulted in adverse clinical outcomes. The present study aimed to determine the expression and prognostic significance of GOLPH3 in human hepatocellular carcinoma (HCC). GOLPH3 expression was examined using western blot analysis of 30 paired samples of HCC and adjacent non-cancerous liver tissues. GOLPH3 expression levels were also assessed using immunohistochemistry in 180 HCC samples and paired controls. In addition, the association of GOLPH3 expression with clinicopathological features and clinical outcome was analyzed. Furthermore, the effect of GOLPH3 on HCC cell proliferation and invasion were determined. Western blot analysis revealed that GOLPH3 expression was significantly elevated in HCC tissue compared with that of the matched adjacent non-cancerous liver tissue. In addition, the results of the immunohistochemical analysis demonstrated that GOLPH3 expression was positively correlated with the Edmondson-Steiner grade $(\mathrm{P}=0.006)$, vascular invasion $(\mathrm{P}=0.002)$ and serum $\alpha$ feto-protein levels $(\mathrm{P}=0.015)$. GOLPH3 expression was found to be an independent factor for predicting the poor overall survival of HCC patients (hazard ratio, 2.01; 95\% confidence interval, 1.26-3.64; $\mathrm{P}=0.025)$. In addition, GOLPH3 silencing inhibited the proliferation, invasion and migration of $\mathrm{HCC}$ cell lines in vitro. In conclusion, the results of the present study demonstrated that high GOLPH3 levels may be a potential biomarker for the poor prognosis of patients with HCC.
\end{abstract}

Correspondence to: Dr Qian Li, Department of Hepatobiliary Surgery, Affiliated Hospital of Weifang Medical University, No. 2428 Yuhe Road, Weifang, Shandong 261031, P.R. China E-mail: liqiandoc@163.com

Key words: hepatocellular carcinoma, golgi phosphoprotein-3, prognosis, biomarker

\section{Introduction}

Worldwide, hepatocellular carcinoma (HCC) is the fifth most prevalent type of cancer and the third most common cause of cancer-associated mortality (1). HCC has been shown to be highly refractory to treatment and has a low five-year survival rate of $\sim 12-15 \%$ worldwide $(2,3,4)$. Early diagnosis is critical for the timely treatment of HCC and improving the survival rate of patients (5). Therefore, it is essential to identify novel biomarkers, which may allow for earlier diagnosis of HCC, provide novel therapeutic targets for HCC treatment and ultimately improve patient survival $(6,7)$.

Golgi phosphoprotein-3 (GOLPH3) is a member of the trans-Golgi matrix family. Previous studies have identified GOLPH3 as an oncogene, which has a role in the development of numerous types of cancer, including lung, ovary, breast, colon and prostate cancer, as well as melanoma, rhabdomyosarcoma and glioma (8-11). In addition, it has been reported that GOLPH3 overexpression promoted cell proliferation and tumorigenesis through activation of the mammalian target of rapamycin (mTOR) signaling pathway, which enhanced AKT activity and decreased forkhead box protein O1 (FOXO1) transcriptional activity $(8,12,13)$. However, studies regarding the correlation between GOLPH3 expression and the prognosis of patients with $\mathrm{HCC}$ are limited. In the present study, immunohistochemical analysis of human HCC and adjacent non-cancerous hepatic tissues was conducted in order to determine the expression of GOLPH3, as well as to investigate whether there was a correlation between GOLPH3 expression and clinicopathological factors associated with HCC prognosis. In addition, HCC cell lines were used to explore the effect of GOLPH3 silencing on cell proliferation, migration and invasion in HCC.

\section{Patients and methods}

Patients and specimens. Paired tissue specimens of HCC and adjacent non-cancerous hepatic tissues (distance from tumor, $\geq 1.5 \mathrm{~cm}$ ) were obtained from 180 patients with primary HCC who underwent surgical resection without pre-operative treatment at the Department of Hepatobiliary Surgery, Affiliated Hospital of Weifang Medical University (Shandong, China) between 2006 and 2008. The participants consisted of 118 male and 62 female patients, with a median age of 52.6 years (range, 34-83 years). In addition to these 180 patients, another group 
consisted of 30 fresh HCC specimens and paired adjacent non-cancerous tissue, which were assessed for GOLPH3 protein expression using western blot analysis. The present study was approved by the ethics committee of the Affiliated Hospital of Weifang Medical University and conformed to the Declaration of Helsinki. Written informed consent was obtained from each patient or their legal guardians. Clinical parameters (as shown in Table I) were obtained by consulting the hospital records. The overall survival was measured as the time from surgery until the patient succumbed to HCC, or the last observation taken. For patients who survived, the data were censored at the last follow-up appointment. Following surgery, patients were followed up every 3 months for the first year, then every 6 months for the subsequent two years and then annually. After surgery the patients received neoadjuvant chemotherapy, radiation therapy or immunotherapy, based on the National Comprehensive Cancer Network recommendations.

Immunohistochemistry (IHC). IHC analysis was performed in order to detect GOLPH3 expression in 180 HCC tissues. Paraffin-embedded specimens (4 $\mu \mathrm{m}$ sections) were administered 3\% hydrogen peroxide in methanol in order to quench endogenous peroxidase activity and then incubated with $1 \%$ bovine serum albumin (Beijing Biosynthesis Biotechnology Co., Ltd., Beijing, China) in order to avoid non-specific binding. Anti-GOLPH3 (1:50, sc-242931; Santa Cruz Biotechnology, Inc., Dallas, TX, USA) was incubated with the sections overnight at $4^{\circ} \mathrm{C}$. Following washing with Tris-buffered saline with Tween-20 (TBS-T) buffer (Beijing Biosynthesis Biotechnology Co., Ltd.), the tissue sections were treated with biotinylated secondary antibodies (sc-2054; Santa Cruz Biotechnology, Inc.), followed by incubation with a streptavidin-horseradish peroxidase complex (Santa Cruz Biotechnology, Inc.). Normal mouse serum (Beijing Biosynthesis Biotechnology Co., Ltd.) was used as a negative control. Tissue specimens were scored by two independent pathologists blinded to the clinical data, using an immunoreactivity scoring system, as previously described (14). In case of discrepancies, a final score was established by reassessment using a double-headed microscope (BH2; Olympus, Tokyo, Japan). The immunostaining for GOLPH3 was semi-quantitatively scored, where only cytoplasmic staining was considered positive, as follows: -, no or $<5 \%$ positive cells;,$+ 5-25 \%$ positive cells;,$++ 26-50 \%$ positive cells; and,$+++>50 \%$ positive cells. For statistical analysis, the scores of ++ and +++ were classified as high expression; and scores of - and + as low expression.

Cell culture. Four HCC cell lines (HCCLM3, MHCC97-H, MHCC97-L and Hep3B) were obtained from the America Type Culture Collection (Manassas, VA, USA) for use in the present study. Cells were cultivated in Dulbecco's modified Eagle's medium (DMEM) supplemented with $10 \%$ fetal calf serum (Sigma-Aldrich, St. Louis, MO, USA). Cells ( $1 \times 10^{5}$ cells/well) were seeded onto six-well cell culture plates for $48 \mathrm{~h}$ until further use.

Western blot analysis. Following surgery the tissues were immediately frozen in liquid nitrogen and stored at $-80^{\circ} \mathrm{C}$ until further use. Frozen tumor sections or cells were lysed in cell lysis buffer (Qiagen, Hilden, Germany). Samples were homogenized and stored at $4^{\circ} \mathrm{C}$ for $30 \mathrm{~min}$. Cell extracts were then subjected to centrifugation at $1,5000 \mathrm{xg}$ for $30 \mathrm{~min}$ at $4^{\circ} \mathrm{C}$ and protein concentration was determined using a bicinchoninic acid assay kit (Qiagen). Total proteins (60 $\mu \mathrm{g})$ from each sample were heated at $98^{\circ} \mathrm{C}$ for 5 min following mixing with SDS loading buffer. Samples were separated using 12\% SDS-PAGE and electrotransferred to polyvinylidene fluoride membranes (Millipore, Billerica, MA, USA). Membranes were blocked with TBS-T containing 5\% skimmed milk at room temperature for $2 \mathrm{~h}$. Membranes were then incubated with goat polyclonal anti-GOLPH3 (1:2,000, sc-242931) or goat polyclonal GAPDH antibodies (1:5,000, sc-20356) (Santa Cruz Biotechnology, Inc.) in TBS-T overnight at $4^{\circ} \mathrm{C}$. Following washing with TBS-T, the membranes were incubated with $5 \%$ skimmed milk in TBS-T buffer, which contained the mouse anti-goat horseradish peroxidase-conjugated immunoglobulin $\mathrm{G}$ secondary antibody (1:30,00, sc-2355; Santa Cruz Biotechnology, Inc.) for $1 \mathrm{~h}$ at room temperature. Proteins of interest were detected and visualized using autoradiography (Beijing Biosynthesis Biotechnology Co., Ltd.) following various exposure times.

Small interfering (si)RNA transfection. HCC cells were transfected with GOLPH3 siRNA or control siRNA (Beijing Biosynthesis Biotechnology Co., Ltd.) using Lipofectamine $2000^{\circledR}$ (Invitrogen Life Technologies) according to the manufacturer's instructions. Cells transfected with siRNA ( $1 \times 10^{5}$ cells/well) were seeded onto six-well plates and cultured for $24 \mathrm{~h}$ prior to harvesting for further analysis. The silencing effect of these siRNAs was detected using western blot analysis, as described previously.

3-(4,5-dimethylthiazol-2-yl)-2,5-diphenyltetrazolium bromide (MTT) assays. Cells ( $4 \times 10^{3}$ cells/well) were plated onto 96-well plates and each well was incubated with $20 \mu 1$ MTT $(5 \mathrm{mg} / \mathrm{ml}$; Sigma-Aldrich) at $37^{\circ} \mathrm{C}$ for $4 \mathrm{~h}$. Absorbance was measured at $490 \mathrm{~nm}$ using a microplate reader. Cells incubated with culture medium were used as the control group. Each sample was assayed in triplicate.

Matrigel invasion assay. Invasion assays were performed using transwell membrane filter inserts $(6.5-\mathrm{mm}$ diameter; $8-\mu \mathrm{m}$ pore; BD Biosciences, Franklin Lakes, NJ, USA) in a 24-well tissue culture plate. Briefly, transfected cells were harvested at $24 \mathrm{~h}$ and resuspended in serum-free RPMI-1640. Cells ( $2 \times 10^{5}$ cells/well) in $200 \mu$ l growth medium without FBS were added to the upper chamber, and the bottom chamber was filled with $500 \mu$ l growth medium containing $10 \%$ FBS. After $48 \mathrm{~h}$, non-migrating cells were removed from the top of the filter with a cotton swab. Invading cells on the bottom of the filter were fixed with methanol, and stained using the Diff-Quick Stain kit (IMEB Inc., San Marcos, CA, USA) according to the instructions. Stained cells in 10 random fields were counted using an inverted microscope (Leica DMI 3000M; Leica, Wetzlar, Germany). Each experiment was conducted in triplicate.

Cell migration assay. The cell migration assays were performed in a chamber system consisting of polycarbonate membrane inserts with an $8-\mu \mathrm{m}$ pore size (Corning, New York, NY, USA) placed in 24-well cell culture insert companion plates. The migration assay was conducted at $48 \mathrm{~h}$ after the cells were 
Table I. Correlation between GOLPH3 expression and clinicopathologic features in cancerous tissue from 180 patients with hepatocellular carcinoma.

\begin{tabular}{|c|c|c|c|c|}
\hline \multirow[b]{2}{*}{ Clinicopathologic variable } & \multirow[b]{2}{*}{ Number of cases } & \multicolumn{2}{|c|}{ GOLPH3 expression } & \multirow[b]{2}{*}{ P-value } \\
\hline & & Low & High & \\
\hline Gender & & & & 0.230 \\
\hline Male & 118 & 48 & 70 & \\
\hline Female & 62 & 26 & 36 & \\
\hline Age (years) & & & & 0.586 \\
\hline$\leq 60$ & 135 & 60 & 75 & \\
\hline$>60$ & 45 & 14 & 31 & \\
\hline Liver cirrhosis & & & & 0.425 \\
\hline Presence & 136 & 59 & 77 & \\
\hline Absence & 44 & 15 & 29 & \\
\hline Capsular formation & & & & 0.861 \\
\hline Presence & 95 & 44 & 51 & \\
\hline Absence & 85 & 30 & 55 & \\
\hline Tumor size & & & & 0.187 \\
\hline$\leq 5 \mathrm{~cm}$ & 89 & 39 & 50 & \\
\hline$>5 \mathrm{~cm}$ & 91 & 35 & 56 & \\
\hline Tumor nodule number & & & & 0.316 \\
\hline Multiple & 104 & 56 & 48 & \\
\hline Solitary & 76 & 18 & 58 & \\
\hline Edmondson-Steiner grade & & & & 0.006 \\
\hline Stage I-II & 99 & 57 & 42 & \\
\hline Stage III-IV & 81 & 17 & 64 & \\
\hline Vascular invasion & & & & 0.002 \\
\hline Absence & 97 & 44 & 53 & \\
\hline Presence & 83 & 30 & 53 & \\
\hline$\alpha$ feto-protein levels & & & & 0.015 \\
\hline$<400 \mu \mathrm{g} / 1$ & 59 & 15 & 44 & \\
\hline$\geq 400 \mu \mathrm{g} / 1$ & 121 & 59 & 62 & \\
\hline
\end{tabular}

GOLPH3, golgi phosphoprotein 3.

infected with siRNA. The cells (in $200 \mu$ l of growth medium without FBS) were placed in the upper chamber and $500 \mu \mathrm{l}$ of growth medium with 5\% FBS was placed in the lower chamber. The cells were incubated at $37^{\circ} \mathrm{C}$ for $24 \mathrm{~h}$. Following the incubation, the insert membranes were fixed with $75 \%$ methanol for $30 \mathrm{~min}$, stained with $0.5 \%$ crystal violet, and counted. The stained cells were counted under the inverted microscope (10 fields per membrane). Each experiment was performed in triplicate.

Statistical analysis. Data were assessed using a paired Student's $\mathrm{t}$-test or one-way analysis of variance for multiple comparisons, and $\chi^{2}$ test for $2 \times 2$ tables was used to compare the categorical data. Survival curves were compared using the Kaplan-Meier method and log-rank test. The Cox proportional hazard model was used for univariate and multivariate analysis in order to explore the effect of GOLPH3 expression on survival. Statistical analysis was conducted using SPSS 16.0 software (Internation
Business Machines, Armonk, NY, USA). P<0.05 was considered to indicate a statistically significant difference.

\section{Results}

Western blot analysis of GOLPH3 expression in HCC tissues. A total of 30 fresh HCC specimens and paired adjacent non-cancerous tissues were examined for GOLPH3 protein expression. The results showed that GOLPH3 expression at the protein level was markedly increased in HCC tissues compared with that of the paired adjacent non-tumor tissues $(\mathrm{P}<0.01$; Fig. 1).

Correlation of GOLPH3 expression with clinicopathological features in HCC patients. Immunohistochemical staining was used to detect GOLPH3 expression in the cytoplasm of HCC tissues (Fig. 2A-D). In addition, Kaplan-Meier analysis indicated that patients with high GOLPH3 expression had a poorer overall survival rate $(\mathrm{P}=0.0014)$ compared with that of 
Table II. Univariate and multivariate analysis of overall survival in 180 patients with hepatocellular carcinoma.

\begin{tabular}{|c|c|c|c|c|c|c|}
\hline \multirow[b]{2}{*}{ Variable } & \multicolumn{3}{|c|}{ Univariate } & \multicolumn{3}{|c|}{ Multivariate } \\
\hline & HR & $95 \% \mathrm{CI}$ & P-value & HR & $95 \% \mathrm{CI}$ & P-value \\
\hline Gender & 0.89 & $0.37-1.25$ & 0.290 & & & \\
\hline Age (years) & 0.71 & $0.22-1.14$ & 0.410 & & & \\
\hline Vascular invasion & 2.18 & $1.14-3.96$ & 0.011 & 1.72 & $0.74-3.16$ & 0.016 \\
\hline No. of nodules & 0.81 & $0.44-1.29$ & 0.190 & & & \\
\hline Cirrhosis & 0.73 & $0.32-1.31$ & 0.360 & & & \\
\hline Serum AFP & 1.06 & $0.47-1.72$ & 0.130 & & & \\
\hline Edmondson-Steiner grade & 1.89 & $1.96-3.70$ & 0.023 & 1.37 & $1.02-2.19$ & 0.476 \\
\hline Vascular invasion & 2.78 & $1.64-5.98$ & 0.001 & 2.29 & $1.02-3.81$ & 0.020 \\
\hline GOLPH3 expression & 3.71 & $1.75-6.11$ & 0.009 & 2.01 & $1.26-3.64$ & 0.025 \\
\hline
\end{tabular}

HR, hazard ratio; CI, confidence interval; AFP, $\alpha$-fetoprotein ( $\leq 400$ vs. $>400 \mathrm{ng} / \mathrm{ml}$ ).

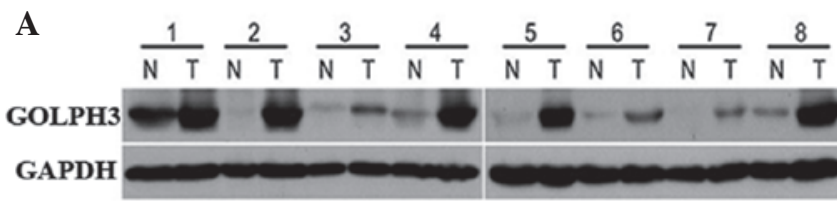

\section{B}

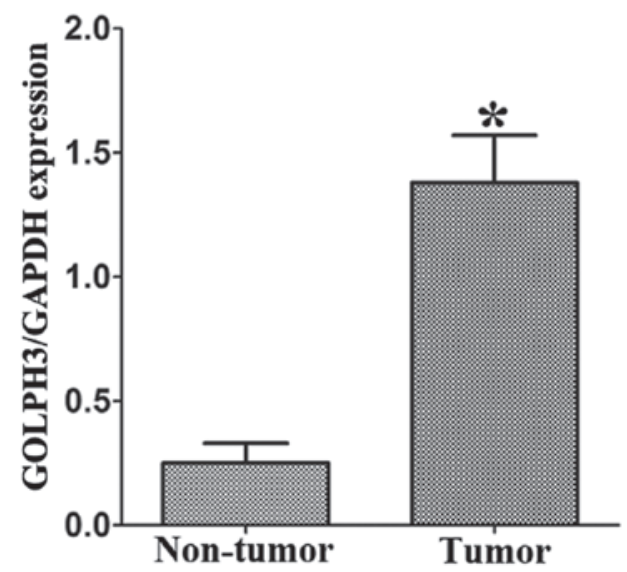

Figure 1. Increased GOLPH3 protein expression in HCC tissue (A) Representative western blots of GOLPH3 protein expression in eight $\mathrm{HCC}$ and adjacent non-cancerous tissues. (B) Relative protein expression of GOLPH3/GAPDH in HCC and non-tumor tissues $(n=30)$. Values are presented as the mean \pm standard deviation. ${ }^{~} \mathrm{P}<0.01$ vs. non-tumor tissue. $\mathrm{N}$, non-tumor tissues; T, tumor tissues; GOLPH3, Golgi phosphoprotein 3; $\mathrm{HCC}$, hepatocellular carcinoma.

patients with low GOLPH3 expression (Fig. 2E). The results revealed that high GOLPH3 expression was observed in 58.8\% (106 of 180) of HCC tissues samples. By contrast, high expression of GOLPH3 was only observed in 7.7\% (14 of 180) of non-cancerous tissues $(\mathrm{P}<0.001)$. In line with the results of the western blot analysis, these data indicated that GOLPH3 expression was significantly upregulated in HCC samples.

As shown in Table I, high GOLPH3 expression was found to be positively correlated with Edmondson-Steiner grade $(\mathrm{P}=0.006)$, vascular invasion $(\mathrm{P}=0.002)$ and serum $\alpha$ feto-protein (AFP) levels $(\mathrm{P}=0.015)$. In addition, univariate and multivariate analyses demonstrated that GOLPH3 was
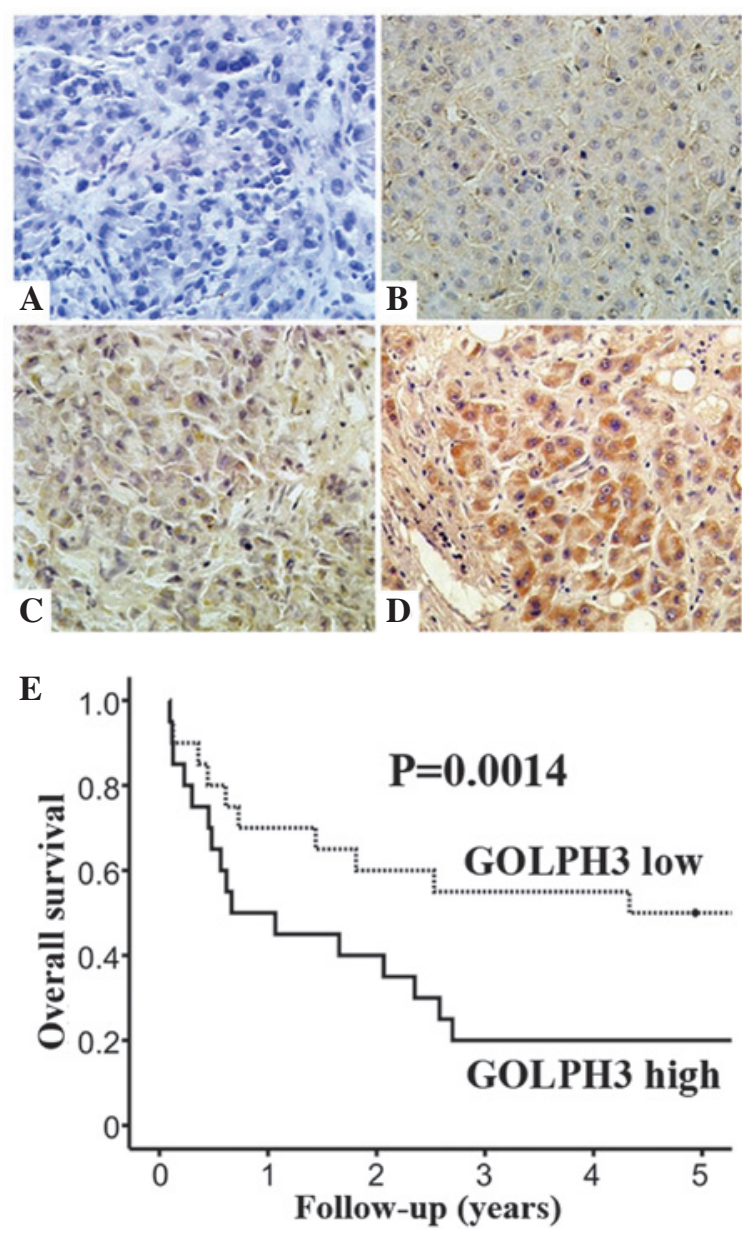

Figure 2. IHC staining of GOLPH3 expression in human HCC tumor tissue. Representative images of (A) negative (-), (B) weak, (C) moderate and (D) strong IHC staining of GOLPH3. Magnification, x200. (E) Kaplan-Meier analysis of overall survival in $180 \mathrm{HCC}$ patients using log-rank test. IHC, immunohistochemical; HCC, hepatocellular carcinoma; GOLPH3, golgi phosphoprotein 3.

an independent prognostic factor for the overall survival of HCC patients (hazard ratio, 2.01; 95\% confidence interval, 1.26-3.64; $\mathrm{P}=0.025$ ) (Table II). 

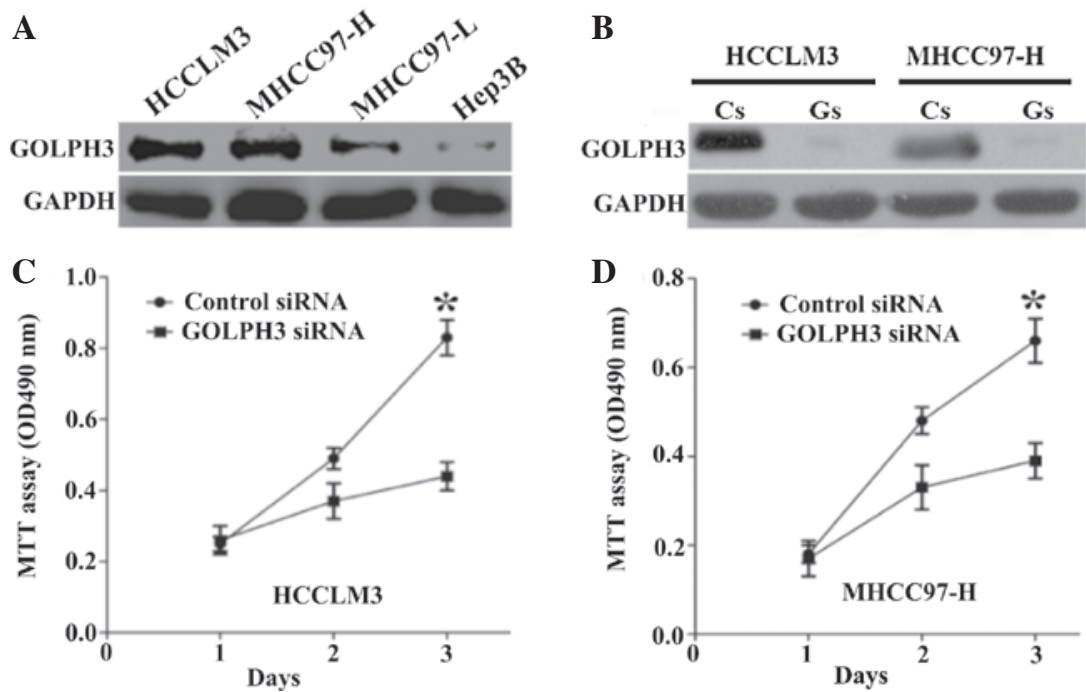

Figure 3. Expression of GOLPH3 in HCC cell lines and the effect of GOLPH3 silencing on HCC cells. (A) Western blot analysis of GOLPH3 protein expression in four HCC cell lines, HCCLM3, MHCC97-H, MHCC97-L and Hep3B. (B) Western blot analysis confirmed the silencing efficacy of GOLPH3 siRNA. MTT assays showed that knockdown of GOLPH3 significantly reduced cell proliferation of (C) HCCLM3 and (D) MHCC97-H cells compared with control siRNA transfected cells. Values are presented as the mean \pm standard deviation. ${ }^{*} \mathrm{P}<0.01$ vs. control siRNA. GOLPH3, Golgi phosphoprotein 3; HCC, hepatocellular carcinoma; siRNA, small interfering RNA; MTT, 3-(4,5-dimethylthiazol-2-yl)-2,5-diphenyltetrazolium bromide; Cs, control siRNA; Gs, GOLPH3 siRNA.
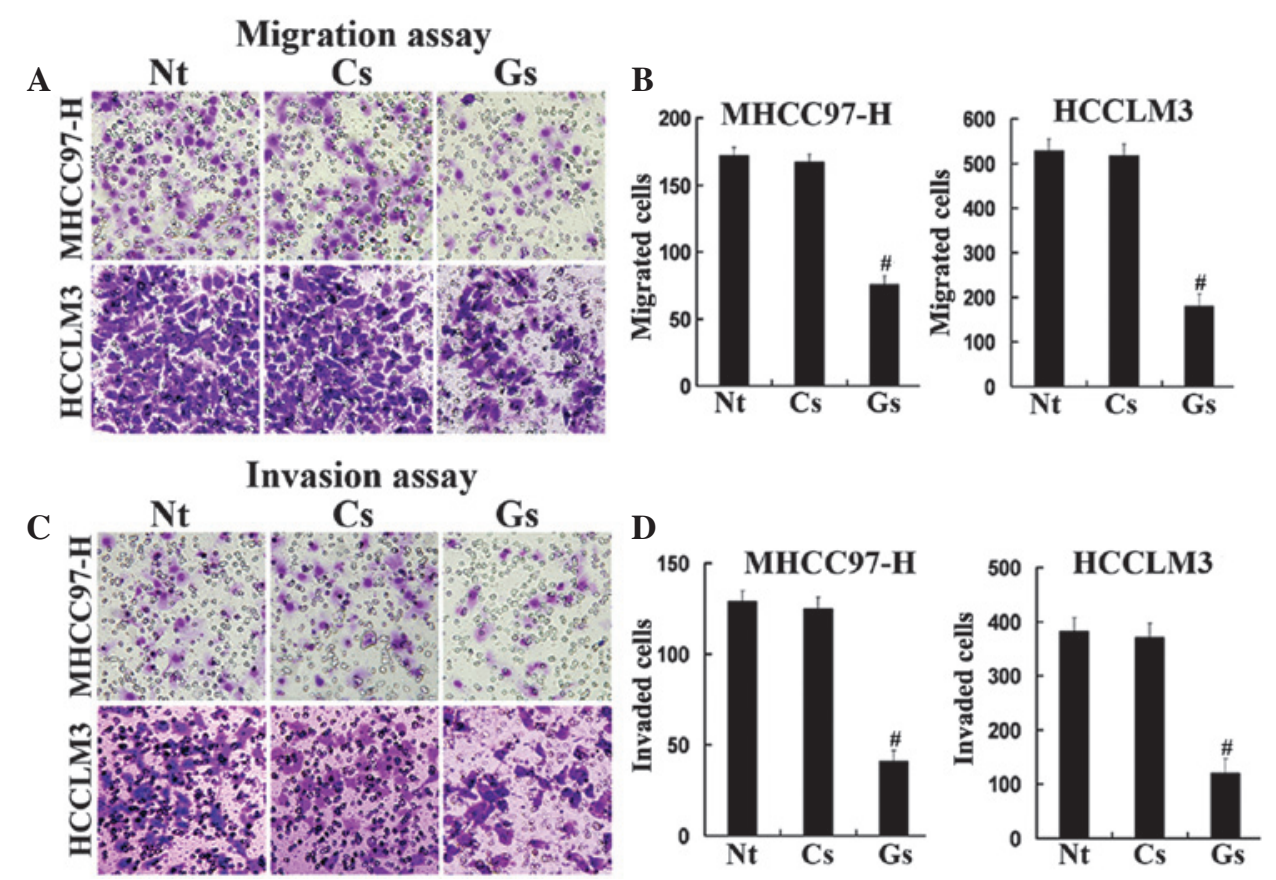

Figure 4. GOLPH3 knockdown inhibits HCC cell migration and invasion of HCCLM3 and MHCC97-H cells in vitro. (A) Representative images (Diff-Quick stain; magnification, $\mathrm{x} 400$ ) and (B) quantitative analysis of the migration of Gs-treated cells analyzed by Transwell assays compared with that of Nt and Cs-treated cells. (C) Representative images (Diff-Quick stain; magnification, x400) and (D) quantitative analysis of the invasive capacity of Gs-treated cells analyzed by Transwell assays compared with that of $\mathrm{Nt}$ and Cs-treated cells. Values are presented as the mean \pm standard deviation. ${ }^{~} \mathrm{P}<0.05$ vs. Cs-treated cells. GOLPH3, Golgi phosphoprotein 3; HCC, hepatocellular carcinoma; siRNA, small interfering RNA; Nt, no treatment group; Cs, control siRNA transfected group; Gs, GOLPH3 siRNA transfected group.

GOLPH3 silencing inhibits the proliferation, migration and invasion of HCC cell lines. Expression levels of GOLPH3 protein were examined in four HCC cell lines, HCCLM3, MHCC97-H, MHCC97-L and Hep3B. HCCLM3 and MHCC97-H cells showed higher GOLPH3 expression compared with that of MHCC97-L and Hep3B cells (Fig. 3A). Therefore, HCCLM3 and MHCC97-H were selected for use in the GOLPH3 silencing experiments.
Western blot analysis confirmed the silencing efficacy of GOLPH3 siRNA (Fig. 3B). In addition, MTT assays demonstrated that the knockdown of GOLPH3 significantly reduced cell proliferation in HCCLM3 and MHCC97-H cells compared with that of the control cells (Fig. 3C and D). Furthermore, GOLPH3 knockdown inhibited hepatocellular carcinoma (HCC) cell migration and the invasion of HCCLM3 and MHCC97-H cells in vitro (Fig. 4). These data indicated 
that GOLPH3 silencing inhibited the proliferation, migration and invasion of HCC cells.

\section{Discussion}

The results of the present study demonstrated that GOLPH3 expression was significantly elevated in HCC tissues compared with that of adjacent non-cancerous liver tissue. In addition, the high expression levels of GOLPH3 were found to have a significant positive correlation with Edmondson-Steiner grade, vascular invasion and serum AFP levels. Furthermore, the association of GOLPH3 expression with prognosis was assessed and it was revealed that GOLPH3 was an independent factor for predicting poor survival in HCC patients.

The GOLPH3 gene was found to be located on chromosome 5 p13 in humans. In addition, this gene was reported to be frequently amplified in several types of solid tumor, including lung, ovary, breast, prostate and skin cancers (8). However, the clinical relevance of GOLPH3 in patients with HCC remained to be fully elucidated. Previous studies have indicated that high expression levels of GOLPH3 promoted tumorigenesis and the progression of several types of malignancies as well as correlated with poor survival rates $(10,14-18)$. One study demonstrated that GOLPH3 expression was present in $>50 \%$ of the patients with glioma and GOLPH3 expression in the glioma was associated with the severity of the tumor (10). In addition, overexpression of GOLPH3 has been associated with the poor prognosis of patients with $\mathrm{cN} 0$ oral tongue cancer; therefore, it was suggested that GOLPH3 may have potential for use as a novel and useful prognostic indicator of $\mathrm{cN} 0$ oral tongue cancer (14). Furthermore, high GOLPH3 expression has been associated with the poor outcome of glioblastoma multiforme patients (15). In vitro siRNA experiments that downregulated GOLPH3 expression resulted in the suppressed proliferation and clonogenic growth in a cultured glioblastoma multiform cell line (15). It was also reported that GOLPH3 overexpression was associated with the transition of prostate cancer from the hormone sensitive phase into the hormone refractory phase; this therefore indicated that GOLPH3 may be an important prognostic indicator for patients with prostate cancer (16). A marked increase in GOLPH3 expression was reported to occur in esophageal squamous cell cancer (ESCC) cell lines and tissues at the mRNA and protein level (17). In addition, high GOLPH3 expression in ESCC was reported to be positively correlated with clinical stage, tumor-node-metastasis (TNM) classification, histological differentiation and vital status; furthermore, the expression of GOLPH3 was shown to be an independent prognostic factor for patients with ESCC (17). It has been demonstrated that the overexpression of GOLPH3 was associated with the size of the tumor, histological grade, depth of invasion, lymph node metastasis, distant metastasis and TNM stage in gastric cancer; in addition, multivariate analysis indicated that GOLPH3 expression levels were an independent prognostic factor for gastric cancer patients following radical surgical resection (18). The results of the present study demonstrated a high GOLPH3 expression rate of 58.8\% in HCC tissues. By contrast, the percentage of patients with high expression of GOLPH3 in non-HCC tissues significantly lower at $7.7 \%(\mathrm{P}<0.01)$. Immunohistochemical analysis statistics demonstrated that high GOLPH3 expression was positively correlated with Edmondson-Steiner grade, vascular invasion and serum AFP levels. Kaplan-Meier analysis indicated that patients with high GOLPH3 expression had a poorer overall survival rate compared with that of patients with low GOLPH3 expression. In addition, univariate and multivariate analyses showed that GOLPH3 was an independent prognostic factor for the overall survival of HCC patients. Furthermore, the results of the present study also suggested that GOLPH3 enhanced the proliferation and invasion of HCC cells.

In conclusion, the results of the present study indicated that GOLPH3 may have an important role in the pathogenesis of human HCC. In addition, GOLPH3 overexpression was found to be a novel prognostic marker for HCC; however, further studies are required in order to explore the underlying mechanisms of action of GOLPH3 in HCC.

\section{References}

1. Knudsen ES, Gopal P and Singal AG: The changing landscape of hepatocellular carcinoma: etiology, genetics, and therapy. Am J Pathol 184: 574-583, 2014.

2. Jemal A, Siegel R, Ward E, Murray T, Xu J and Thun MJ: Cancer statistics, 2007. CA Cancer J Clin 57: 43-66, 2007.

3. Salgia R and Singal AG: Hepatocellular carcinoma and other liver lesions. Med Clin North Am 98: 103-118, 2014.

4. Bosetti C, Turati F and La Vecchia C: Hepatocellular carcinoma epidemiology. Best Pract Res Clin Gastroenterol 28: 753-770, 2014.

5. Finn RS: Current and future treatment strategies for patients with advanced hepatocellular carcinoma: role of mTOR inhibition. Liver Cancer 1: 247-256, 2012.

6. Song P1, Gao J, Inagaki Y, Kokudo N, Hasegawa K, Sugawara Y and Tang W: Biomarkers: Evaluation of screening for and early diagnosis of hepatocellular carcinoma in Japan and China. Liver Cancer 2: 31-39, 2013.

7. Shao YY, Hsu CH and Cheng AL: Predictive biomarkers of antiangiogenic therapy for advanced hepatocellular carcinoma: Where are we? Liver Cancer 2: 93-107, 2013.

8. Scott KL, Kabbarah O, Liang MC, Ivanova E and Anagnostou V: GOLPH3 modulates mTOR signalling and rapamycin sensitivity in cancer. Nature 459: 1085-1090, 2009.

9. Kunigou O, Nagao H, Kawabata N, Ishidou Y and Nagano S: Role of GOLPH3 and GOLPH3L in the proliferation of human rhabdomyosarcoma. Oncol Rep 26: 1337-1342, 2011.

10. Li XY, Liu W, Chen SF, Zhang LQ, Li XG and Wang LX: Expression of the Golgi phosphoprotein-3 gene in human gliomas: a pilot study. J Neurooncol 105: 159-163, 2011.

11. Romanuik TL, Wang G, Holt RA, Jones SJ, Marra MA and Sadar MD: Identification of novel androgen-responsive genes by sequencing of LongSAGE libraries. BMC Genomics 10: 476, 2009.

12. Abraham RT: GOLPH3 links the Golgi network to mTOR signaling and human cancer. Pigment Cell Melanoma Res 22: 378-379, 2009.

13. Zeng Z, Lin H, Zhao X, Liu G, Wang X, Xu R, Chen K, Li J and Song L: Overexpression of GOLPH3 promotes proliferation and tumorigenicity in breast cancer via suppression of the FOXO1 transcription factor. Clin Cancer Res 18: 4059-4069, 2012.

14. Lin L, Qin Y, Jin T, Liu S, Zhang S, Shen X and Lin Z: Significance of NQO1 overexpression for prognostic evaluation of gastric adenocarcinoma. Exp Mol Pathol 96: 200-205, 2013.

15. Li H, Guo L, Chen SW, Zhao XH, Zhuang SM, Wang LP, Song LB and Song M: GOLPH3 overexpression correlates with tumor progression and poor prognosis in patients with clinically N0 oral tongue cancer. J Transl Med 10: 168, 2012.

16. Zhou J, Xu T, Qin R, Yan Y, Chen C, Chen Y, Yu H, Xia C, Lu Y, Ding X, Wang Y, Cai X and Chen J: Overexpression of Golgi phosphoprotein-3 (GOLPH3) in glioblastoma multiforme is associated with worse prognosis. J Neurooncol 110: 195-203, 2012.

17. Hua X, Yu L, Pan W, Huang X, Liao Z, Xian Q, Fang L and Shen H: Increased expression of Golgi phosphoprotein-3 is associated with tumor aggressiveness and poor prognosis of prostate cancer. Diagn Pathol 7: 127, 2012.

18. Wang JH, Chen XT, Wen ZS, Zheng M, Deng JM, Wang MZ, Lin HX, Chen K, Li J, Yun JP, Luo RZ and Song LB: High expression of GOLPH3 in esophageal squamous cell carcinoma correlates with poor prognosis. PLoS One 7: e45622, 2012. 